\title{
IMPLEMENTACIÓN DE UN SISTEMA DE ALIMENTACIÓN ININTERRUMPIDA, CON VISUALIZACIÓN DE PARÁMETROS ELÉCTRICOS EN LCD
}

Franklin Giovanni Parra

Tecnólogo en Electricidad

Universidad Distrital Francisco José de Caldas

frankgpf999@hotmail.com

Bogotá, Colombia

\section{Jorge Alberto Obregón Merchán}

Tecnólogo en Electricidad

Universidad Distrital Francisco José de Caldas

jaobregonmrc@hotmail.com

Bogotá, Colombia

\section{César Augusto Hernández Suárez}

Magister en Ciencias de la Información y las Comunicaciones

Docente planta de la Universidad Distrital Francisco José de Caldas cahernandezs@udistrital.edu.co Bogotá, Colombia

Tipo: Artículo de reflexión

Fecha de Recepción: Febrero 25 de 2013

Fecha de Aceptación: Mayo 2 de 2013

\section{IMPLEMENTATION OF AN UNINTERRUPTIBLE POW- ER SUPPLY WITH ELECTRICAL PARAMETER DISPLAY LCD}

\begin{abstract}
This paper presents the design and implementation of an uninterruptible power supply (UPS) with display of electrical parameters (voltage, current and power) in a liquid crystal display (LCD), performed by logical-mathematical processes scheduled on a microcontroller, to provide constant power to a critical load, and in turn to know the behavior of the electrical variables of the device that needs to be fed.
\end{abstract}

Key words: critical charge, electric power quality, ITI curve, UPS.

\section{RESUMEN}

Este artículo presenta el diseño y la implementación de un sistema de alimentación ininterrumpida (UPS) con visualización de parámetros eléctricos (tensión, corriente y potencia) en una pantalla de cristal líquido (LCD), realizado mediante procesos lógico-matemáticos programados en un microcontrolador, con el fin de brindar alimentación constante a una carga crítica y a su vez conocer el comportamiento de las variables eléctricas del dispositivo que requiera ser alimentado.

Palabras claves: calidad de la potencia eléctrica, carga crítica, curva ITI, UPS 


\section{INTRODUCCIÓN}

Se puede definir una UPS (uninterrumpible power supply) como un equipo o dispositivo capaz de suministrar potencia o energía frente a alguna interrupción de lo que sería el suministro normal de la misma. Además puede agregar otras funciones que terminan mejorando el suministro de energía eléctrica a los equipos sofisticados o de alto riesgo eléctrico que tiene conectados a ella. Entre las cosas que agrega puede contarse un estabilizador de la energía eléctrica entrante, aislación de la fuente de energía de eléctrica normal, filtrado de la energía entrante, corrección de la forma de onda, corrección de la frecuencia de línea, protección a periféricos de las CPU o incluso sus partes, como placas de red o modem's, monitoreo de la energía de línea, para optimizar la protección, etc. Puede darse que el agregado de funciones genere distintos tipos o topología de construcción de estos equipos.

La proliferación de equipos electrónicos altamente sensibles a las fluctuaciones de voltaje, la liberación de los agentes del mercado y las expectativas incrementadas a los clientes hace de la CPE una necesidad imperativa. Este asunto ha tomado considerable importancia en los últimos años debido al aumento de interrupciones de procesos productivos en las industrias, ya que ellas utilizan equipos y controles extremadamente sensibles a las variaciones de la tensión, generando impactos económicos sobre los consumidores y las empresas de servicios. Los problemas de la calidad de la energía eléctrica incluyen una gran variedad de disturbios electromagnéticos, que pueden tener su origen dentro o fuera de las instalaciones de los clientes o las empresas de servicios, pudiendo causar muchos tipos de efectos no deseados en las cargas, generando insatisfacción en el cliente, el cual con su nueva posición en un mercado desregulado, puede tomar decisiones trascendentales que llegan hasta demandas contra las empresas de servicios; luego es una imperiosa necesidad el obtener niveles adecuados de la calidad de la energía eléctrica de modo que se logre una satisfacción del cliente y una conservación del mercado por parte de la empresa de servicios.

Las UPS corrigen los errores de la red, los cuales hacen parte del estudio de calidad de la potencia, sin embargo, resulta conveniente saber cómo es el comportamiento de la energía eléctrica una vez que es corregida por la UPS al ser entregada a una carga, con el fin de tener una mayor confiabilidad del desempeño tanto de la UPS como del dispositivo que está conectado a ella; en este artículo vemos como se implementa el diseño de una UPS a la cual se le adiciona una pantalla de cristal líquido que muestra visualmente algunos parámetros de la calidad de la potencia.

\section{FUNDAMENTOS}

Una UPS es una fuente de suministro eléctrico que posee una batería con el fin de seguir dando energía a un dispositivo en el caso de interrupción eléctrica. Los UPS son llamados en español SAI (Sistema de alimentación ininterrumpida). Las UPS suelen conectarse a la alimentación de las computadoras, permitiendo usarlas varios minutos en el caso de que se produzca un corte eléctrico. Algunos UPS también ofrecen aplicaciones que se encargan de realizar ciertos procedimientos automáticamente para los casos en que el usuario no esté y se corte el suministro eléctrico.

\subsection{Tipos de UPS}

SPS (stand by power systems) u off-line: un SPS se encarga de monitorear la entrada de energía figura 1, cambiando a la batería apenas detecta problemas en el suministro eléctrico. Ese pequeño cambio de origen de la energía puede tomar algunos milisegundos [4].

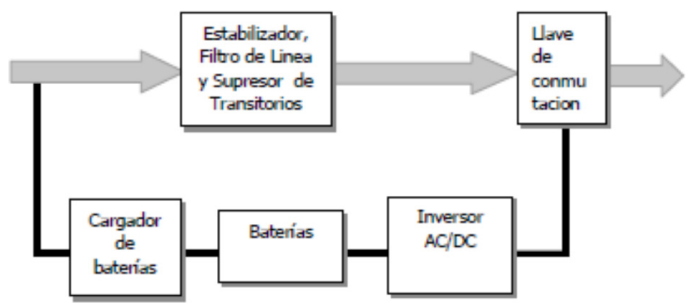

Figura 1. Esquema básico de una UPS interactiva operando con el suministro de la red. 
UPS on-line: Evita esos milisegundos sin energía al producirse un corte eléctrico figura 2, pues provee alimentación constante desde su batería y no de forma directa. El UPS on-line tiene una variante llamada by-pass [4].

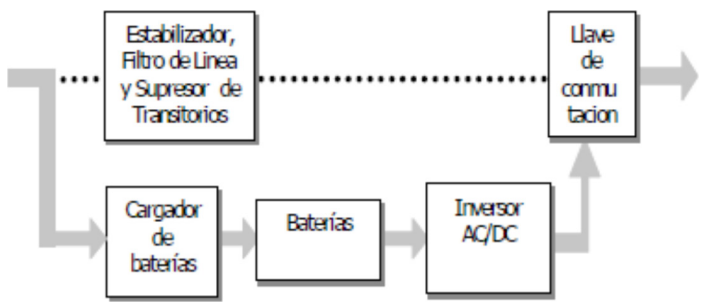

Figura 2. Esquema básico de una UPS on-line operando con el suministro de la red.

\subsection{Componentes típicos de las UPS}

Rectificador: rectifica la corriente alterna de entrada, proveyendo corriente continua para cargar la batería. Desde la batería se alimenta el inversor que nuevamente convierte la corriente en alterna. Cuando se descarga la batería, ésta se vuelve a cargar en un lapso de 8 a 10 horas, por este motivo la capacidad del cargador debe ser proporcional al tamaño de la batería necesaria.

Batería: se encarga de suministrar la energía en caso de interrupción de la corriente eléctrica. Su capacidad, que se mide en Amperes Hora, depende de su autonomía (cantidad de tiempo que puede proveer energía sin alimentación).

Inversor: transforma la corriente continua en corriente alterna, la cual alimenta los dispositivos conectados a la salida del UPS.

Conmutador (By-Pass) de dos posiciones, que permite conectar la salida con la entrada del UPS (By Pass) o con la salida del inversor.

Actualmente, los sistemas de alimentación ininterrumpida o UPS, brindan una gran confiabilidad y efectividad al funcionamiento de cargas críticas cuyo trabajo requiera operación constante sin que haya parada de procesos, pero en algunos casos, es conveniente e interesante conocer el comportamiento de la carga en cuanto a condiciones eléctricas, de modo que para una UPS convencional, la inclusión de un analizador de red resultaría bastante costoso y complejo.

La mayoría de las ocasione las UPS alimentan cargas especificas puntuales, conocidas también como cargas criticas como algunos aparatos médicos, elementos industriales en donde se requiera operación continua de un proceso, elementos informáticos y elementos de comunicaciones; en donde podemos deducir que, éstos elementos necesitan alimentación constantemente y además que la energía que reciben sea de buena calidad. Claro está, que estos dispositivos están diseñados para que operen ante fallas emergentes de corta duración, es decir, entre unos 5 a 25 minutos, aunque de acuerdo a características de diseño se pueden encontrar algunos UPS que tengan una duración de operación autónoma de hasta varias horas, pero ese aspecto está determinado por la capacidad que dispongan las baterías.

\subsection{Calidad de la potencia}

Esencialmente en los sistemas de red eléctrica existe una gran cantidad de problemas que pueden llegar a causar daños en los equipos que se encuentren conectados, estas falencias en la red hacen parte del estudio de calidad de la potencia que los dispositivos UPS pueden llegar a corregir, A continuación se mencionan los problemas mas comunes tenidos en cuenta:

- Fallas en la potencia: definido como la pérdida total de tensión de entrada.

- Sobretensiones o SWELL: son incrementos momentáneos o prolongados en la tensión principal.

- Hundimientos de tensión o SAG's: son reducciones momentáneas o prolongadas de la tensión principal.

- Picos: son sobre tensiones en pequeños períodos a frecuencia y tensión mayores al nominal.

- Ruido: es una frecuencia alta u oscilación transitoria, generalmente producida por otros equipos cercanos a la red de suministro local.

- Inestabilidad de la frecuencia: definido como pequeños cambios en la frecuencia principal de la red, $50 / 60 \mathrm{~Hz}$. 
- Distorsión armónica: es la desviación de la onda sinoidal ideal esperada en la línea.

La resolución CREG 016 del año 2007, establece controles para los operadores de red en donde se exige rangos de parámetros de operación y el suministro eficiente de la energía en cuanto a las diferentes fallas que puedan surgir. Estos parámetros están contemplados en el estudio de la calidad de la potencia [7].

Entre los parámetros mencionados, encontramos los hundimientos de tensión (SAG), una de las fallas mas frecuentes en la figura 3, y las elevaciones de tensión (SWELL), los cuales son desviaciones de tensión que pueden ser corregidas por UPS.

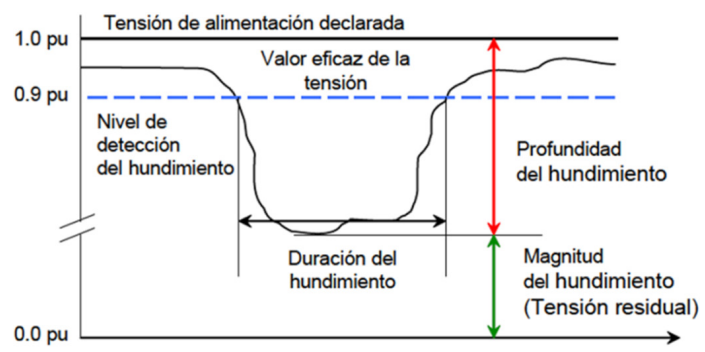

Figura 3. Descripción de un hundimiento de tensión SAG.

La curva ITI (information technology industry) describe una envolvente de la tensión de alimentación la cual puede ser típicamente tolerada por la mayoría de equipos de tecnología de información (ETI) es decir que no causa salida de operación de este tipo de equipos [6], [7].

Esta curva es aplicable para sistemas eléctricos de tensión nominal a $60 \mathrm{~Hz}$. Otras tensiones nominales no son consideradas específicamente y es responsabilidad del usuario determinar la aplicabilidad de esta curva para otras aplicaciones.

Al observar la figura 4 se aprecia una zona que está delimitada por dos curvas (superior e inferior).

En esta zona de operación "segura" del equipo, la zona en la que no se afecta la continuidad del proceso (para combinaciones determinadas de magnitud y duración). Las combinaciones de magnitud y duración que estén por debajo de la curva inferior, se constituyen en los SAG's que afectan la continuidad del proceso [6].

Existen dos formas tradicionales en que las UPS pueden corregir estos factores: el de topología híbrida, que su funcionamiento se basa entre el uso interactivo de la red y la energía proveniente de una batería, realizando una compensación a la red; por otro lado, se encuentra la UPS de línea interactiva, en donde su modo de operación consiste en hacer suplencias completas entre la red y la energía proporcionada por un banco de baterías.

A continuación se presenta el desarrollo de un modelo de UPS que trabaja mediante el sistema de línea interactiva y adicionalmente muestra las variables eléctricas presentes en la carga.

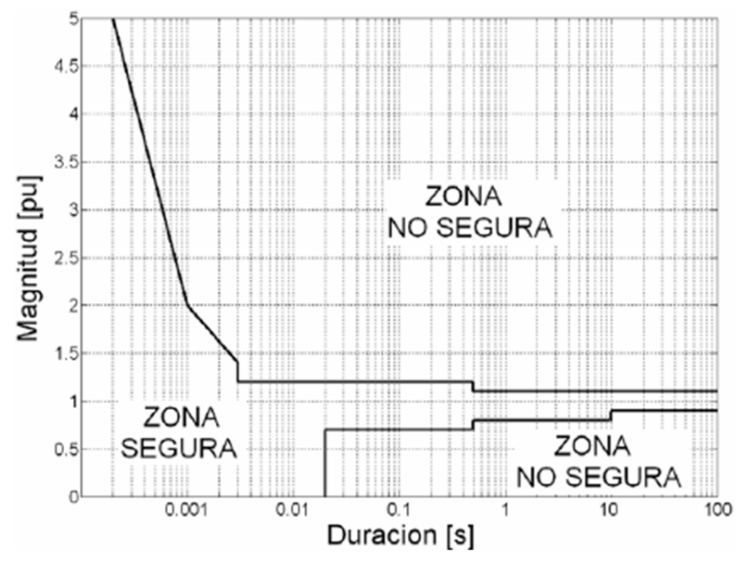

Figura 4. Descripción la curva ITI.

\section{METODOLOGÍA}

Para realizar el diseño de la UPS, es necesario inicialmente determinar las condiciones eléctricas a las cuales se desea operar, tales como tensión de alimentación, potencia a suministrar, forma de la señal, autonomía de la batería (tiempo de respaldo), esquema de operación y esquema de protecciones. para este caso se ha determinado una tensión de operación de 120 $\mathrm{V}$ y una potencia nominal de $250 \mathrm{VA}$, a partir de estas condiciones se establecen las características eléctricas de los materiales necesarios, de igual manera es importante determinar también la forma de trabajo de la ups ya sea on-line 
o interactiva, es decir que entre en operación en el momento en el que detecte una falla, o que esté operando constantemente con la red local, para el diseño desarrollado se ha tenido en cuenta un tipo de operación interactiva.

Para el desarrollo del prototipo es necesario trabajarlo en pequeños grupos, dentro de los cuales cada uno constituye una función determinada.

El sistema general de una UPS que se diseñó, se muestra en el diagrama de bloques de la figura 5, cada elemento del diagrama tiene sus respectivos componentes internos y funciones trascendentales; a continuación serán explicados cada uno de los bloques del diagrama anteriormente mencionado.

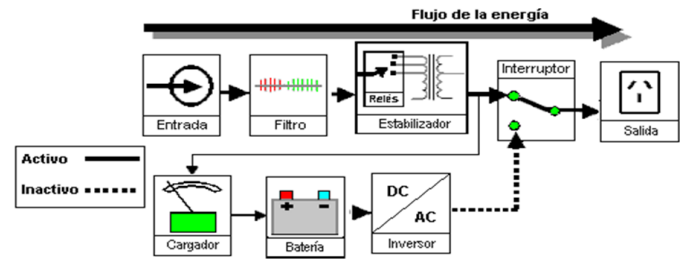

Figura 5. Descripción de operación UPS desarrollada.

\subsection{Detector de componente}

El elemento inicial, que es el detector de componente, es el elemento encargado de controlar el interruptor de entrada para el funcionamiento del UPS; se le ha denominado detector de componente ya que es el encargado de detectar la presencia de tensión alterna correspondiente a la entrada del sistema general, además, tiene en cuenta los tiempos y las tensiones de respuesta de operación de los equipos con respecto a la curva ITI, si la tensión es muy alta, hay en elemento a la entrada (varistor) del sistema, que produce un corte en la tensión excesiva, si la tensión es demasiado baja, el detector de componente acciona el interruptor de entrada y opera la batería central para que la carga crítica no tenga parada de procesos; una vez el servicio de red es restablecido a los valores nominales, se acciona el interruptor de entrada principal y sale de servicio la batería. En la figura 6, se puede observar el esquema del circuito utilizado en el detector de componente.

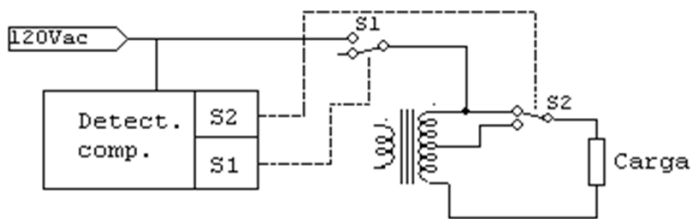

Figura 6. Modelo de operación del detector de componente.

\subsection{Cargador de batería}

Uno de los elementos esenciales para el correcto funcionamiento de una UPS, es el cargador de baterías; cada entrada de funcionamiento de la UPS con la baterías hace necesaria la recarga del banco de baterías principal. Las baterías son cargadas a una tensión entre 13,4-13,8 V, por lo general en todas las UPS tienen baterías de 12V. Se utilizó un modelo mediante pulsos, que ayuda a conservar la vida útil de las baterías y además se aprovechan las interrupciones para medir el nivel de carga de la batería. Todo el esquema del cargador de baterías se puede ver en la figura 7.

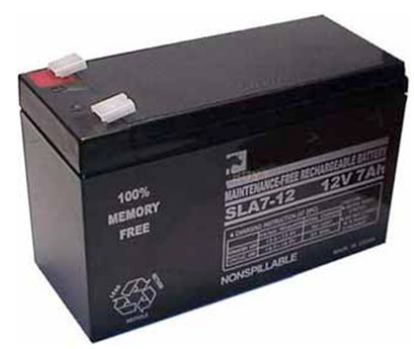

Figura 7. Modelo regular de batería recargable usadas en UPS's convencionales.

\subsection{Batería principal}

La batería es la fuente suplementaria del sistema general, es la encargada en entregar la energía en caso de algún corte de energía o dado que se presente un SAG, pero el manejo de las baterías recargables pueden llegar a ser bastante dispendiosas, ya que muchas de las características mencionadas en la placa, no son tan correctas como nos gustaría, además, las baterías tienen una vida útil bastante limitada, dependiendo de la frecuencia de uso, carga y descarga, modo de carga, energía exigida por la carga y el tiempo de autonomía requerida, que son algunos de los parámetros de mayor aten- 
ción, por lo tanto, se hace necesario hacer uso del cálculo de la autonomía de las baterías.

Los fabricantes de baterías recargables entregan un valor de la capacidad de la batería representados en el nivel de tensión, que es el nivel de tensión nominal de la batería, y entregan un valor en amperios-hora, que en teoría estos datos representan la capacidad de corriente que la batería puede entregar por un período de una hora; pero en realidad esto no es así, si se asume esto y se aplica, podemos estar poniendo en juego la duración de la vida útil de la batería. Para esto se ha determinado una ecuación que hace posible calcular la autonomía de un banco de baterías recargables, y está descrita como la ecuación (1).

$$
t=\frac{H}{\left(\frac{I H}{c}\right)^{k}}
$$

Donde:

t: tiempo de autonomía.

C: capacidad en Ah

$\mathrm{H}$ : capacidad de la batería con respecto al tiempo en que está calculada la capacidad nominal (este dato por lo general no está en los datos impresos de la batería, pero por lo general es 20 h aún en el peor de los casos).

I: corriente que será exigida a la batería.

k: exponente de Peukert (es un valor relacionado al tipo de materiales de la batería, si es de baterías de gel es 1,1, y si es de ácido de plomo es de 1,3, a menos que el fabricante entregue otro dato).

Con respecto a esto, los datos de la batería que se adquirió son los siguientes:

Nivel de tensión: $12 \mathrm{~V}$.

Capacidad: 7,0Ah.

Tipo de batería: ácido de plomo.

Relación de tiempo: desconocida, por lo tanto, se asume $20 \mathrm{~h}$.

De acuerdo a los datos anteriores se puede deducir que para una carga de $10 \mathrm{~A}$, el tiempo de autonomía se obtiene mediante la ecuación (2).

$$
t=\frac{20}{\left(\frac{10 \cdot 20}{7}\right)^{1,3}}
$$

0,2561 horas, que equivale a 15 minutos aproximadamente cuando la batería se encuentre en buen estado.

\subsection{El inversor}

Se podría decir que el inversor es uno de los elementos de mayor importancia y de bastante complejidad de un sistema de alimentación ininterrumpida; aunque solo es el encargado de convertir corriente continua en corriente alterna, sin este elemento una UPS sería inútil.

Existen una gran variedad de circuitos inversores, que pueden entregar señales cuadradas, triangulares o sinusoidales, en donde éstas últimas son las más complejas de desarrollar (figura 8 y 9).

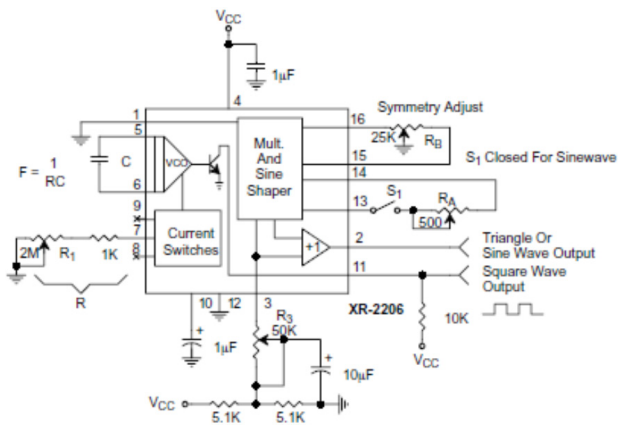

Figura 8. Esquema de conexión del CI XR2206 para generar onda sinusoidal con la mínima distorsión armónica.

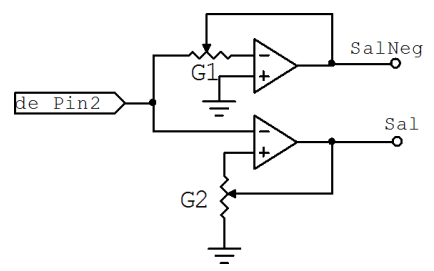

Figura 9. Amplificador buffer para la salida del pin 2 desde el CI XR-2206.

Aunque se podría hacer uso de circuitos tanque RL o de redes RC retroalimentadas para generarlas, se hace dispendioso el uso de ellas cuando se requieren señales de baja frecuencia 
debido a que se producen con una distorsión muy alta y no son muy estables, además que los valores de las impedancias de los elementos no se consiguen fácilmente o en su defecto no existen, las UPS por lo general utilizan inversores que entregan a la salida una señal cuadrada o escalonada figura 10 , pero aunque resulte más sencillo, sin embargo, con señales cuadradas la presencia de armónicos es mas evidente y más difícil de controlar, el inversor que se desarrolló, entrega una señal sinusoidal con un THD de aproximadamente $1.5 \%$, dependiendo de las características exteriores de temperatura. La figura 11, muestra el esquema circuital del inversor, que utiliza como elemento principal el CI XR2206 que es un generador de precisión monolítico, puede entregar a la salida una señal cuadra, y una señal triangular o sinusoidal, de acuerdo a la forma en que sea conectado, para nuestro caso, se conecta para que entregue a la salida una señal sinusoidal a una frecuencia de $60 \mathrm{~Hz}$.

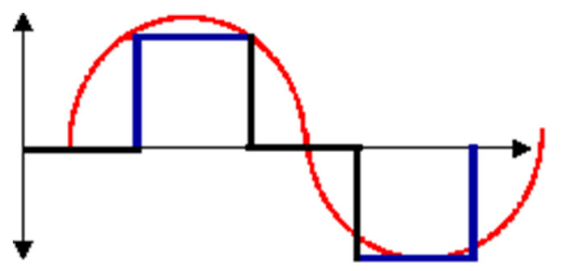

Figura 10. Forma de onda entregada por una UPS de tipo off-line o interactiva.

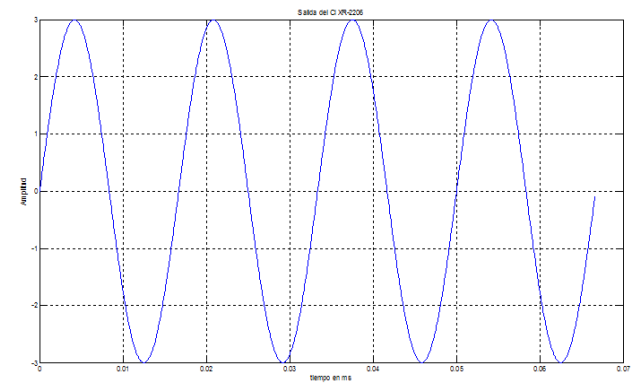

Figura 11. Forma de onda generada por el CI XR-2206 configurado a $60 \mathrm{~Hz}$.

Dado que es un generador monolítico, se hace necesario acoplar a la salida unos dos preamplificadores mediante el CI TL072, el primero que amplifique la señal en amplitud y el otro que tome esa misma señal y la invierta, finalmente se tendrán dos señales, una sinusoidal y la otra también sinusoidal pero desfasada $180^{\circ}$. Es necesario tener estas dos señales por separado, porque se requieren al momento de energizar el transformador principal, que es quien entrega la energía a valores nominales de tensión.

La frecuencia de salida del circuito para cualquier tipo de señal está dada por los valores de R y C por la relación en ecuación (3).

$$
f_{o}=\frac{1}{R C}[H z]
$$

En donde R es la resistencia equivalente conectada al terminal 7, y C es la capacitancia total en los terminales 5 y 6 . Para lograr un frecuencia de $60 \mathrm{~Hz}$, asumimos una capacitancia aleatoria de $2.2 \mu \mathrm{F}$, por lo tanto nos daría una resistencia de $7575,76 \Omega$, que sabemos muy bien que este valor de resistencia es imposible conseguirla en el mercado, por lo tanto se acopla una resistencia de $1 \mathrm{k} \Omega$ en serie con un trimmer ajustable de $10 \mathrm{k} \Omega$, en donde se hace el respectivo ajuste de la frecuencia para lograr una mayor exactitud. El potenciómetro R3, se utiliza para ajustar la amplitud a la salida del XR-2206, que de acuerdo a los valores de la ganancia de los amplificadores se ajusta para que no se produzcan distorsiones.

\subsection{Amplificador de potencia}

Uno de los criterios más importantes en un sistema electrónico de potencia es que el acoplamiento entre las señales de entrada y las de salida, tengan una relación salida-entrada que se pueda ajustar figura 12 .

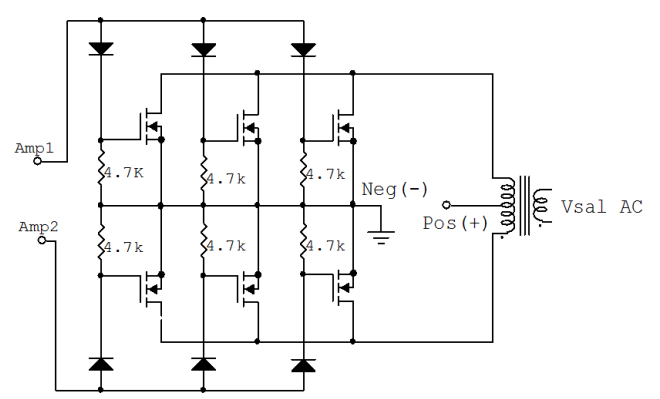

Figura 12. Amplificador de potencia acoplado al transformador de salida para la carga crítica. 
El propósito de la etapa de potencia de este circuito es producir una ganancia en corriente y tensión a la salida, especialmente de corriente, para que la potencia entregada a la carga sea suficiente para hacer funcionar un equipo de la forma más cercana a la nominal.

\subsection{Protección y medida}

En todo sistema de suministro de potencia, siempre es necesario hacer uso de protecciones, aunque el uso en baja tensión no es muy crítico, pero se deben utilizar para evitar daños en la carga, o en la fuente o al personal que manipule los equipos. Las protecciones requeridas para este sistema son bastante sencillas de aplicar, ya que no son muy exigentes, pero sin embargo se debe tener mucho cuidado al momento de configurarlas. Las protecciones que se utilizaron son: protección termo-magnética y protección diferencial. Generalmente, existen sistemas de protecciones en baja tensión que pueden integrar muchas funciones en un solo dispositivo, es el caso de los tomacorrientes tipo GFCI, que integran todas las protecciones mínimas requeridas. Luego, se hace el acoplamiento de un dispositivo de estos para hacer el complemento de los equipos de protección contra cortocircuito, sobrecarga y diferencial.

\subsection{Adquisición y publicación de las mediciones realizadas}

Para realizar la visualización de los parámetros eléctricos en la LCD, es necesario llevar las muestras de tensión y corriente en el punto de acople entre la carga y la UPS, a una de las entradas del microcontrolador, para esto es necesario atenuar los valores reales mediante transformadores de corriente (TC) y transformadores de tensión (TP), luego de ser atenuadas y adaptadas, se envían a un microcontrolador, el trabajo del microcontrolador será en primera medida, pasar los datos que recibe de tensión y corriente por un conversor análogodigital, los datos recibidos los almacena en una matriz en una memoria externa EEPROM (figura 13); teniendo en cuenta las condiciones de período de la señal y de intervalos de tiempo constantes, los datos capturados por los con- versores $A D$ se procesan mediante métodos lógico-matemáticos para determinar la tensión y la corriente en valores RMS presentados en la carga [8], teniendo en cuenta que los valores RMS de una señal está dada por la ecuación (4) [5].

$$
X_{r m s}=\sqrt{\frac{1}{T} \int_{-T / 2}^{T / 2} x^{2} d t}
$$

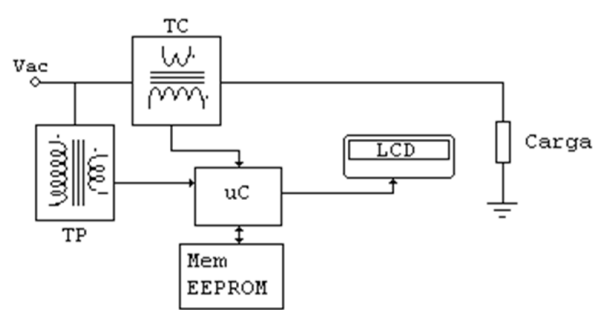

Figura 13. Modelo general para la captura y visualización de datos.

Con esos datos ya almacenados, se hace el respectivo análisis para el cálculo de la potencia, sabiendo que para la potencia promedio como ecuación (5) es [5].

$$
P_{\text {prom }}=\frac{1}{T} \int_{-T / 2}^{T / 2} p(t) d t
$$

Debido a que las señales capturadas están de forma discreta, es necesario hacer el uso de métodos y modelos matemáticos, como los métodos de integración de Simpson, series de potencias y aproximaciones numéricas [1].

Finalmente, cuando ya se han hecho los respectivos procesos para el cálculo de cada una de las diferentes variables, se muestran en la LCD. Mediante un pulsador sencillo, se puede navegar a través de cada una de las variables.

\section{ANÁLISIS DE RESULTADOS}

Se realizaron diferentes tipos de pruebas al prototipo para asegurar el correcto funcionamiento tanto del dispositivo como de la carga crítica conectada, para esto se realizaron las pruebas que se mencionan a continuación.

Se realizó prueba de capacidad de potencia, y se puede asegurar que la capacidad de sumi- 
nistro de potencia es de al menos unos 250VA. Para esto, se le conectaron cargas diferentes tipos, que requirieron diferentes cantidades de potencia, los resultados fueron bastante satisfactorios hasta una potencia de 300VA, pero para condiciones de garantía y seguridad, se estableció que la potencia nominal de salida fuera de 250VA.

Se realizaron pruebas de la señal de onda generada, para asegurar que la entrega de la señal a la salida fuera una señal sinusoidal con un nivel de distorsión armónica (THD) $\geq 1,5 \%$.

Se realizaron pruebas al sistema integral de adquisición de datos, para la publicación de los valores de medida. Se utilizaron divisores de tensión para la medida de la tensión y se utilizaron transformadores especializados para medición de la corriente. Los resultados obtenidos fueron bastante precisos con una tolerancia de $\pm 15 \%$ para diferentes tipos de cargas en condiciones de falla de la red.

Se realizaron pruebas tiempos de respuesta ante falla de modo que cumplieran con las normas basadas en la tabla ITI (figura 4), los resultados fueron aceptables con una respuesta ante falla de $66 \mathrm{~ms}$.

La prueba de autonomía de la batería a potencia nominal es de 12 minutos, teniendo en cuenta que la batería estuviera en estado útil óptimo, pero se sabe que el tiempo de autonomía de la batería puede disminuir con el uso.

Se realizaron pruebas en las protecciones en/y para la carga, las protecciones utilizadas son: sobrecarga, cortocircuito y diferencial; los ajustes se realizaron para que funcionara durante 10 minutos con una sobrecarga de $15 \%$ por encima de la potencia nominal, para cortocircuito se utilizó una respuesta de tiempo inverso para que actuara ante fallas de cortocircuito, y finalmente para la protección diferencial, se le hicieron ajustes para que actuara con un porcentaje de corriente de fuga de $7 \%$; eso es de aproximadamente $30 \mathrm{~mA}$. Todo esto se integró de forma exitosa mediante un acoplamiento de un tomacorriente tipo GFCI.
Se realizaron las pruebas correspondientes a las alarmas. Las alarmas indican sobrecarga, trabajo con batería y batería baja. Para la alarma de sobrecarga, se sometió el equipo a una potencia de 270VA, en donde al registrar la sobrecarga, se identifica una alarma audible constante y visual mediante un anuncio lumínico; en la alarma correspondiente al trabajo con baterías, se ajustó para que produzca un sonido con una intermitencia de $5 \mathrm{~s}$, y finalmente para el ajuste de la alarma por batería baja, se ajustó para produjera un sonido con intermitencia constante.

\subsection{Especificaciones técnicas}

\section{Entrada}

Tensión monofásica: 120 VAC $\pm 10 \%$

Frecuencia: $60 \mathrm{~Hz}+/-5 \%$

Factor de potencia: $>0,92$

Eficiencia: mayor a 0,85

\section{Salida}

Potencia: $250 \mathrm{VA}$ - $230 \mathrm{~W}$

Tensión: $120 \mathrm{VAC}+/-5 \%$ para cargas nominales lineales, $+/-10 \%$ para cargas no lineales

Frecuencia: $60 \mathrm{~Hz}+/-0,1 \mathrm{~Hz}$

Forma de Onda: Sinusoidal

Distorsión (THD) < 1.5 \% para carga no lineal

Sobrecarga: $108 \%$ para 10 minutos

\section{Generales}

Tipo de batería: 12V, 7Ah

Display de LCD

Ambientales

Temperatura: $4^{\circ}$ a $43^{\circ} \mathrm{C}$

Ruido audible: $55 \mathrm{~dB}$ a 1 metro de distancia

Tomas de Salida

Tipo: GFCI (ground fault circuit interrupter).

\section{CONCLUSIONES}

Se ha logrado diseñar y construir un sistema de alimentación ininterrumpida (UPS), que es capaz de entregar una potencia de 250VA, con una señal sinusoidal con un THD aproximado de 1,5\%, entrega una tensión eficaz de 115 Vrms a carga nominal y una autonomía de 10 minutos; los sistemas de control utilizados fueron tanto analógicos como digitales con unos 
tiempos de respuestas que cumplen con los requerimientos generales de los dispositivos críticos; al mismo tiempo, se logró la visualización de los parámetros eléctricos en un LCD que fueron tratados mediante un microcontrolador.

De acuerdo a lo desarrollado, se puede concluir que una UPS puede incluir más funciones a parte de las básicas, como la visualización de

\section{Referencias Bibliográficas}

[1] R. Nagle; Ecuaciones diferenciales y problemas con valores en la frontera, cuarta edición, México: Pearson educación, 2005.

[2] J. Ferreira; Microcontroladores: programación, familias y sus distintas aplicaciones en la industria, primera edición, Colombia: Alfa-omega, 2007.

[3] E. E. Staff-M.I.T, Circuitos magnéticos y transformadores, Massachusetts: Reverté, 2003.

[4] C-MOS, UPS. Estabilizadores de tensión, Protección Inteligente, pp. 2-6 los parámetros eléctricos. También es posible hacer que una UPS entregue señal sinusoidal al momento de usar la batería interna como fuente principal.

Diferencia de la mayoría de las UPS disponibles en el mercado, se logró diseñar una que entregara señal sinusoidal con un THD bastante reducido, menor al 1,5\%.

[5] M. Sadiku; Fundamentals of electrical circuits, third edition, New York: Mc Graw-Hill, 2004.

[6] Varios, Norma Técnica Colombiana NTC 5001, parámetros de calidad de la potencia, Colombia, ICONTEC, 2008.

[7] Resolución CREG024 de 2005, Ministerio de Minas y Energía, normas de la calidad de la potencia, Colombia, 2005.

[8] E. García; Compilador C CCS y simulador Proteus para microcontroladores PIC, primera edición, España: Alfa-omega, 2008. 